\title{
Dispensación excepcional: una necesidad del paciente
}

\section{Eduardo Satué de Velasco}

Vicepresidente $2^{\circ}$ de SEFAC. Farmacéutico comunitario en Maella (Zaragoza).

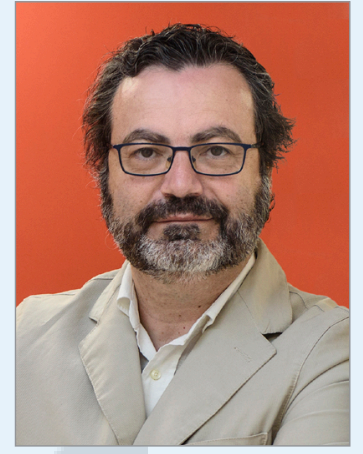

Eduardo Satué de Velasco

\section{PALABRAS CLAVE}

Prescripción

complementaria,

dispensación excepcional, servicios colaborativos

\section{KEYWORDS}

Supplementary prescription, exceptional dispensation, collaborative services
Cualquier farmacéutico comunitario es consciente de que el proceso prescripción-dispensación en España está plagado de lagunas e ineficiencias que la pandemia de la COVID-19 no ha hecho más que poner en mayor evidencia si cabe. Lo que llama la atención de todo esto son las escasas competencias que asigna la ley al farmacéutico comunitario para ayudar al paciente a seguir su tratamiento ante el más nimio fallo formal en la prescripción. Estas competencias quedan reducidas a la sustitución de genérico bioequivalente a otro con exactamente la misma presentación, dosis, forma galénica etc. y como algo excepcional la posibilidad de bloquear la dispensación en caso de riesgo evidente para la salud. Esta situación es claramente insuficiente en el día a día donde encontramos prescripciones incompletas, desabastecimientos frecuentes, formas galénicas inadecuadas, extravíos, roturas... y todo tipo de situaciones en las que la única solución oficial para que el paciente pueda acceder a su tratamiento es: "vaya usted al centro de salud", algo que si bien era una dificultad, con la actual situación de centros de salud con acceso limitado y teléfonos colapsados es a veces misión imposible. En la práctica esto supone que o bien el paciente consigue la intervención del médico en un plazo razonablemente corto (antes de que se le termine la medicación) o bien lo usa de forma inadecuada o bien la interrumpe con las consecuencias que ello pueda acarrearle en términos de empeoramiento del problema de salud e incluso del abandono definitivo del tratamiento. Recordemos que esto no es un problema que tenga el farmacéutico comunitario sino el paciente, y que está originado por el sistema sanitario, que no le presta a esta circunstancia la debida atención.

Los farmacéuticos comunitarios podemos alertar de esta situación, que queda a menudo en un segundo plano ante otros temas también urgentes. Sí existen iniciativas autonómicas de gran éxito, plasmadas en los convenios de colaboración entre la Administración y los Colegios Oficiales de Farmacéuticos, que contemplan actuaciones que se añaden a las antes descritas, tal y como sucede en Aragón, donde existe la posibilidad de que el farmacéutico intervenga en situaciones de urgencia o falta de suministro para suplir, en ciertos casos, un medicamento por otro similar. Un avance sin duda que debería extenderse al resto del país dentro de lo que ya se conoce como "dispensación excepcional"; esto es, la posibilidad de salvaguardar el espíritu de la prescripción por parte del farmacéutico, ante una situación de urgencia o necesidad, en la que dispensa el medicamento más adecuado dentro de las posibilidades reales de las que dispone con el fin de garantizar al paciente su disponibilidad y uso adecuado de su medicación de acuerdo con el paciente y sus necesidades. Esta realidad está regulada ya desde hace años en muchos países desarrollados como Reino Unido, Canadá, EEUU, Australia, Nueva Zelanda... y apoyada desde hace años por la Federación Internacional Farmacéutica (FIP).

Sin embargo, la actuación del farmacéutico comunitario no termina en la dispensación excepcional, que si bien es importante no cubre el abanico de posibilidades que puede ofrecer al paciente, prescriptores y al sistema sanitario. La dispensación excepcional solo sería una parte de lo que denominamos "prescripción complementaria", entendida como la posibilidad de adecuar la medicación prescrita por el médico al paciente. no por una situación excepcional que puede conllevar una interrupción o mal uso del medicamento, sino desde el enfoque del trabajo colaborativo como forma habitual de relación entre profesionales sanitarios que velan conjuntamente por la mejora de calidad de vida del paciente (no debemos confundir esta disciplina con la "prescripción independiente" en la que la actuación farmacéutica es independiente del médico). 
La prescripción complementaria amplia las responsabilidades del farmacéutico y necesariamente la coordinación con el prescriptor, ya que debe existir un acuerdo previo sobre las situaciones y medicamentos en los que intervendría el farmacéutico. En este sentido, en la práctica farmacéutica las situaciones pueden ir desde la más sencilla, como puede ser adecuar una forma galénica a la necesidad del paciente, hasta la más compleja, como sería la delegación por parte del médico, tal como sucede en EEUU o Reino Unido, en la instauración del tratamiento en el paciente, dentro de los términos de acuerdo que hayan pactado. Por ejemplo, en EEUU un médico puede realizar un acuerdo con un farmacéutico para que en aquellos pacientes con Infecciones del Tracto Urinario (ITU) que previamente acepten, éste pueda dispensarles antibióticos idóneos sin necesidad de acudir a consulta. 0 subiendo un peldaño, para realizar el ajuste de la medicación en pacientes con antihipertensivos, quedando el farmacéutico responsable de ese seguimiento y de informar periódicamente al médico de los resultados obtenidos.

A escala internacional existen diversos estudios y metanálisis que concluyen la equivalencia de resultados en salud de la prescripción complementaria con la realizada por los médicos en problemas de salud como la hipertensión, hemoglobina glicada, LDL, adherencia al tratamiento, así como en la satisfacción del paciente y su calidad de vida (1). Por otra parte, esta nueva práctica cuenta con la aceptación de los profesionales farmacéuticos (2). Todo ello redunda en una optimización de los recursos sanitarios y sobre todo, en una mejora de la atención sanitaria al paciente que dispone de más y mejores recursos para tratar su salud.

Una vez mostradas las diferentes posibilidades del farmacéutico ante una prescripción, queda en evidencia que la realidad española está muy alejada de la práctica en otros países desarrollados de nuestro entorno. Parece necesario que así como en otros países el propio sistema sanitario ha regulado exitosamente esta práctica para dar soluciones a los problemas habituales que surgen en la prestación farmacéutica a los pacientes, adoptemos soluciones similares en nuestro Sistema Nacional de Salud. En este sentido, desde SEFAC venimos demandando la regulación de la dispensación excepcional como un primer paso para la mejora asistencial del paciente. Por esta razón en este número de la revista disponéis de un artículo sobre un caso práctico de dispensación excepcional, y próximamente se publicará otro artículo sobre un proyecto piloto impulsado por SEFAC en esta materia.

Finalmente, la receta electrónica puede ser el medio natural de intervención y comunicación directa del farmacéutico comunitario con el médico y el ámbito donde hacer efectiva la integración de la farmacia comunitaria en el Sistema Nacional de Salud, evitando filtros innecesarios y circuitos indirectos que pueden lastrar la asistencia al paciente. Esperamos que dada la casuística generalizada experimentada durante la pandemia, la Administración sea consciente al fin de este problema del paciente y lo solvente con el concurso de los farmacéuticos comunitarios como profesionales más propicios para su resolución.

\section{Referencias bibliográficas}

1. Weeks G, George J, Maclure K, Stewart D. Non-medical prescribing versus medical prescribing for acute and chronic disease management in primary and secondary care. Cochrane Database of Systematic Reviews. 2016, Issue 11. Art. No.: CD011227. doi:10.1002/14651858.CD011227.pub2

2. Mills T, Patel N, Ryan K. Pharmacist non-medical prescribing in primary care. A systematic review of views, opinions, and attitudes. International Journal of Clinical Practice. 2020. 0-2 p. doi:10.1111/ijcp. 13827 